\title{
Primeras Disecciones Anatómicas con Fines Docentes en Chile
}

\author{
First Anatomical Dissections for Teaching Purposes in Chile
}

\author{
Juan Silva ${ }^{1,2,3}$; Claudia Araya ${ }^{1,4}$; Julio Cárdenas²; Barbara Mansilla $^{1}$; Matías Salazar ${ }^{1}$ \& Alexis Latin ${ }^{1}$
}

\begin{abstract}
SILVA, J.; ARAYA, C.; CÁRDENAS, J.; MANSILLA, B.; SALAZAR, M. \& LATIN, A. Primeras disecciones anatómicas con fines docentes en Chile. Int. J. Morphol., 39(1):143-150, 2021.
\end{abstract}

RESUMEN: En Chile, los primeros antecedentes de la enseñanza de la anatomía aparecen en el siglo XVI, para proveer conocimientos a los profesionales sanitarios que ejercían en el territorio. En 1704, el Papa Sixto IV permite la práctica de la disección, encabezada por el alcalde de la ciudad y en donde se invitaba a médicos y cirujanos. Posteriormente, la enseñanza exclusivamente teórica de la medicina en la Universidad Real de San Felipe estimuló a los profesores a enseñarla clínica y la anatomía en forma práctica para complementar las clases expositivas. La Independencia de Chile, determinó que las carreras universitarias sufrieran cambios curriculares importantes, aunque en medicina y anatomía, su desarrollo fue lento y tortuoso. El objetivo de este trabajo fue describir las prácticas de la disección anatómica en la formación de médicos en Chile durante el periodo comprendido entre los años 1704y 1889. Se realizó una revisión bibliográfica en libros de historia de Chile, de historia de la medicina chilena y artículos científicos relacionados al tema, esto nos permitió describir los inicios, desarrollo y características de la práctica de la disección utilizada en la formación de médicos en el periodo en cuestión. El análisis de los resultados nos permitió identificar 5 etapas distintas entre sí, fuertemente influidas por su contexto histórico y diferenciadas por el enfoque que les infundieron los profesores de cada una de estas etapas, que definieron una bibliografía, una orientación y una práctica de la disección en función de las formaciones académicas de cada uno de ellos. Este trabajo, permitió ordenar y exponer información valiosa para la historia de la medicina en Chile, y muy importante, comprender las prácticas educativas que se llevaron a cabo en la formación de estos profesionales que tuvieron un rol preponderante en el proceso de establecimiento de la naciente nación.

PALABRA CLAVE: Historia de Chile, historia de la medicina, historia de la anatomía, disección anatómica.

\section{INTRODUCCIÓN}

En Chile durante el periodo histórico comprendido entre los últimos años de la Conquista española y las primeras décadas de la República independiente (1540 a 1863), se llevó a cabo no solo la organización política de la nación, sino también el nacimiento y desarrollo cultural y educacional de la misma. En relación con la educación médica y específicamente la enseñanza de la anatomía es posible observar sus primeros pasos en la segunda mitad del siglo XVI. Ejemplo de esto es que en 1566 el Cabildo de Santiago nombra al Licenciado Don Alonso de Villalobos como asesor y Examinador de Cirugía del Reino de Chile, éste es el primer paso que se daba en el sentido de exigir ciertos conocimientos anatómicos mínimos a los profesionales que ejercían en el territorio. Más tarde en 1704, el mismo organismo tomaba en consideración el Decreto Papal de Sixto IV (italiano 1414-1484, sacerdote franciscano) y acuerda "que se hiciera anatomía del cuerpo humano". A esta práctica encabeza- da por el propio alcalde de la ciudad, se invitaba a médicos y cirujanos (Ferrer, 1904; Flores, 1933; Laval, 1964). Posteriormente la educación en Chile vive un gran adelanto con el establecimiento de la Universidad Real de San Felipe (USF) en 1756. En ella y a cargo del Dr. Domingo Nevín McHugh (irlandés 1722-1770, médico), comienza en 1758 la enseñanza de la medicina universitaria en el país, y pese a que la enseñanza era principalmente teórica, todos los profesores que enseñaron en esta casa de estudios procuraron enseñar la parte práctica de la carrera en el Hospital San Juan de Dios (HSJD) (Laval 1964; Cárdenas, 2006; Laval \& Duarte, 2016). Una vez independizada la República de Chile de España, comenzó la reorganización de la sociedad. En el ámbito educacional, se concreta el cierre de la USF y se inaugura el Instituto Nacional (IN) en su reemplazo. Evidentemente, las carreras universitarias también sufren cambios curriculares importantes, aunque en medicina, y en la

\footnotetext{
${ }^{1}$ Facultad de Medicina y Ciencia, Universidad San Sebastián. Chile.

${ }^{2}$ Departamento de Anatomía y Medicina Legal, Facultad de Medicina. Universidad de Chile. Chile.

${ }^{3}$ Centro de Investigación en Educación, Universidad Bernardo O`Higgins. Chile.

${ }^{4}$ Programa de Especialidad de Ortodoncia y Ortopedia dento-maxilo-facial. Escuela de Graduados. Facultad de Odontología. Universidad de Chile. Chile.
} 
enseñanza de la anatomía, su desarrollo fue lento y tortuoso. El objetivo de este trabajo fue describir las prácticas curriculares formales e informales de la disección anatómica en la formación de médicos en Chile durante el periodo comprendido entre los años 1704 y 1889 , información valiosa para la historia de la medicina en Chile, y muy importante para comprender las prácticas educativas que se llevaron a cabo en la formación de estos profesionales que tuvieron un rol preponderante en el proceso de creación de la naciente nación.

\section{MATERIAL Y MÉTODO}

Una vez definido el problema a investigar se consultaron diversas fuentes de información entre libros de historia de Chile y de la historia de la medicina chilena, artículos científicos relacionados al tema y artículos periodísticos de época (Biblioteca Nacional). Basado en esta literatura, se realizó una revisión exhaustiva en búsqueda de los antecedentes históricos que permitieran describir los inicios, desarrollo y características de la práctica de la disección anatómica utilizada en la formación de médicos desde los inicios de la educación médica formal en la USF y hasta la inauguración de la Escuela Médica de calle San Francisco. En esta investigación histórica, se dio especial importancia a las evidencias que permitieran relacionar, periodos históricos na- cionales, profesores y estudiantes de medicina del periodo, las características de los espacios físicos donde se llevaron a cabo las actividades académicas, la práctica anatómica propiamente tal, la bibliografía utilizada en estos cursos y algunos otros datos de interés.

\section{RESULTADOS Y DISCUSIÓN}

Los resultados obtenidos del estudio de este periodo histórico permiten identificar 5 etapas consecutivas y diferenciadas por influencias externas, sus académicos, su bibliografía y sus prácticas educativas (Tabla I).

Primera etapa (1704-1810). Desde las últimas décadas de la Colonia y hasta la Primera Junta de Gobierno de 1810: caracterizada por médicos y cirujanos latinos y romancistas. La educación era de tipo maestro - aprendiz, validada por el Protomédico (Neghme, 1988). En 1704, el Cabildo acordó que, “... será conveniente que se haga anatomía del cuerpo humano, con asistencia de médicos y cirujanos". En estas prácticas se seguían las indicaciones de Mondino de Luzzi (italiano 1270 - 1326, médico, cirujano y anatomista) (Ferrer; Laval, 1964), y se podían practicar hasta 8 disecciones al año, los pocos conocimientos obtenidos iban dirigidos a la formación de cirujanos (Flores). En 1758 comienza a dictarse el curso de Prima Medicina en la USF

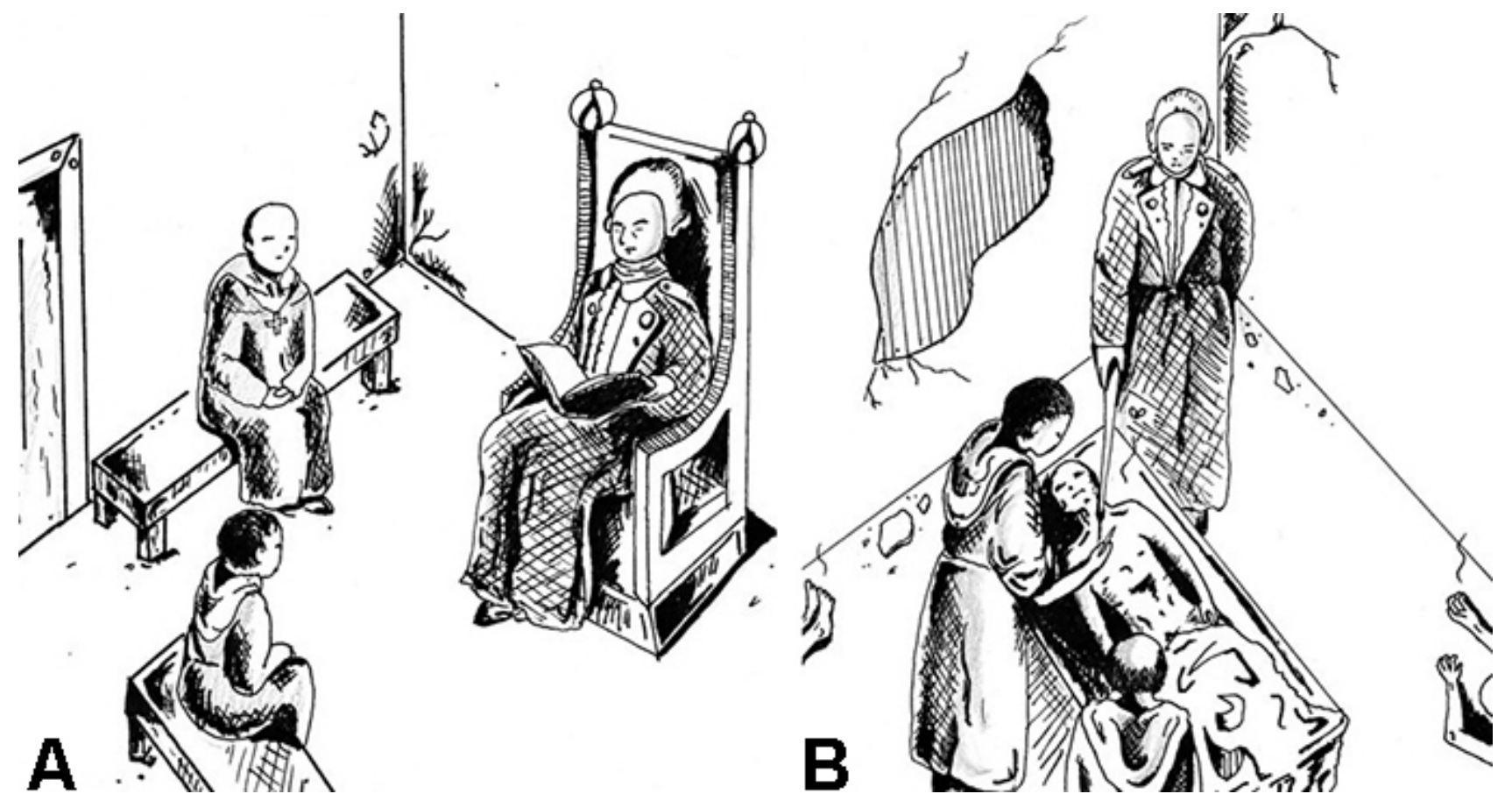

Fig. 1. a) Dr. Domingo Nevín McHugh en una clase de Prima Medicina en la USF. b) El mismo Dr. Nevín, realizando una clase práctica en el depósito de cadáveres del HSJD. En ambas imágenes, se observa como estudiante, al Fray Pedro Manuel Chaparro (representación de los autores según relatos históricos, basado en la portada de “Anatomia Corporis Humani” de Mondino de Luzzi, 1316). 
Tabla I. Resumen de los resultados obtenidos del análisis histórico del periodo de 1704 a 1889. En ella es posible observar en cada una de las etapas descritas en el texto, el periodo de tiempo en que se desarrollo cada una, los profesores del periodo, el lugar físico donde se llevaron a cabo las clases teóricas, la práctica de la disección y la bibliografía recomendada en cada periodo.

\begin{tabular}{|c|c|c|c|c|c|}
\hline Etapa & Años & Profesor(Es) & Clases teóric as & Práctica de la disección & Bibliografía \\
\hline $1^{\text {era }}$ & $1704-1810$ & $\begin{array}{l}\text { Nevín - Zambrano - de los Ríos } \\
\text { - Oliva - Chaparro }\end{array}$ & U. Real de San Felipe & Hospital San Juan de Dios & Heister, 1755 - Martínez, 1764 \\
\hline $2^{\mathrm{da}}$ & $1810-1833$ & Grajales - Cox - Morán & $\begin{array}{l}\text { U. Real de San Felipe, Escuela } \\
\text { Privada de Cox y Morán }\end{array}$ & $\begin{array}{l}\text { Hospital San Juan de Dios - } \\
\text { Cementerio (Panteón) de }\end{array}$ & $\begin{array}{l}\text { López, } 1750 \text { - Bonells y Lacaba } \\
1820\end{array}$ \\
\hline $3^{\text {era }}$ & $1833-1839$ & $\begin{array}{l}\text { Morán, P. - Avello - Morán, B. } \\
\text { - Cox - Sazié }\end{array}$ & Instituto Nacional & $\begin{array}{l}\text { Santiago } \\
\text { Hospital San Juan de Dios }\end{array}$ & $\begin{array}{l}\text { Chaussier, } 1823 \text { - Bichat, } 1829 \\
\text { Maygrier, } 1820 \text { - Tiedemann, } \\
1827\end{array}$ \\
\hline $4^{\text {ta }}$ & $1840-1850$ & Lafargue - Pretot - Padín & Hospital San Juan de Dios & Hospital San Juan de Dios & Lauth, 1841 - Cruveilhier, 1834 \\
\hline $5^{\mathrm{ta}}$ & $1850-1889$ & Aguirre & $\begin{array}{l}\text { Escuela Médica de calle San } \\
\text { Francisco }\end{array}$ & $\begin{array}{l}\text { Escuela Médica de calle San } \\
\text { Francisco }\end{array}$ & Jamain, 1853 \\
\hline
\end{tabular}

a cargo del Dr. Domingo Nevín McHugh quien se limitó a leer y discutir los textos de Hipócrates, Galeno y Avicena (Fig. 1.a), pero informalmente, complementó esta enseñanza con la práctica anatómica tanto en el depósito de cadáveres del HSJD (Fig. 1.b), como también en su cementerio, preferentemente en los meses de invierno (Ferrer; Medina, 1928; Laval 1964; Cárdenas, 2006; Laval \& Duarte). A la muerte del Dr.Nevín, sus sucesores (Dres. Zambrano, de los Ríos y Oliva), continuaron con la tradición de formar en la teoría y en la práctica, a los escasos estudiantes de medicina de la USF. Durante este periodo el Fray Pedro Manuel Chaparro (chileno 1745 - 1811, fraile juandediano y médico), complementaba los estudios médicos de la USF también en el HSJD y reclamaba que no era decente verse obligado a realizar la disección anatómica, en camposanto (Laval, 1964).

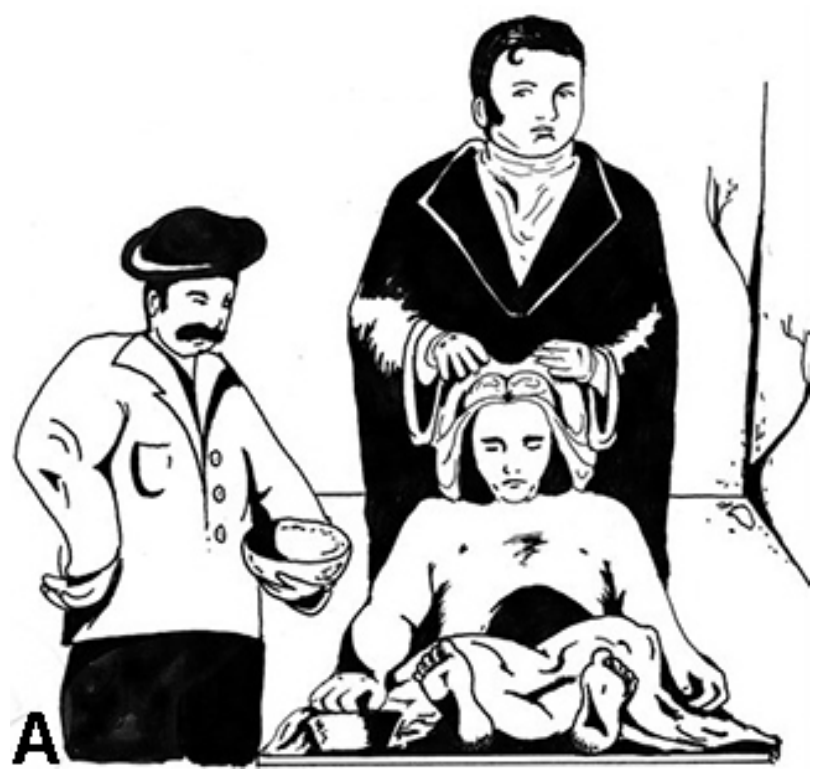

Segunda etapa (1810-1833). Desde la Primera Junta de Gobierno de 1810 y hasta la inauguración de la Escuela Médica del IN en 1833 (EM33): durante este periodo, la formación médica tanto en la USF, como en Escuelas Privadas continuó. Así en 1817, el Dr. Eusebio Oliva (chileno i? 1830, médico) profesor de la USF, quién tuvo un alumno, Domingo Amunategui Muñoz (chileno 1798 - 1842, abogado). Lamentablemente después de un periodo corto de tiempo, abandonó dicha carrera (Benavente, 1928). En 1814 llega al país el Dr. Agustín Nathaniel Miers Cox Lloyd (ingles 1785 - 1869, cirujano y médico), quien, infundido por la tradición inglesa de formación anatómica y quirúrgica en Escuelas Privadas, comenzó a preparar médicos y cirujanos romancistas (Neghme). Más tarde circa de 1819, el Dr. Cox indicaba que “... ya es todo un teatro anatómico el HSJD (Fig. 2.a), en el depósito de cadáveres, hago yo continua-

Fig. 2. a) El Dr. Nathaniel Miers Cox, enseñando anatomía en el depósito de cadáveres del HSJD a cirujanos romancistas, de su Escuela Privada (representación de los autores según relatos históricos, basado en "La Lección de Anatomía del Dr. Deijman”, obra de Rembrandt, 1656). b) El Dr. Manuel Julián Grajales, en sus labores de disección anatómica, mientras formaba cirujanos romancistas en el Panteón de Santiago (representación de los autores según relatos históricos). 
mente disección de ellos, con auxilio de la gran acequia de agua corriente que tiene; y en la misma, aunque algo sombrío y poco ventilado, tengo en maceración los huesos de un hombre para la formación de un esqueleto" (Flores; Laval, 1964). En 1819, se propuso al Director Supremo, Don Bernardo O’Higgins Riquelme (chileno 1778 - 1842, militar y político) al Dr. Manuel Julián García Grajales Gil de la Sierra (español 1775 - 1855, cirujano y médico) como Profesor de Anatomía y Cirugía (Fig. 2.b). El Dr. Grajales comentaba en 1825 , que “....apenas hay un día que no haga disecciones; pero es lo más triste tener que hacerlas a descubierto sobre las miasmas de los sepulcros, tostado por el sol en el verano y sobre el barro en el invierno"(del Panteón de Santiago hoy Cementerio General de Santiago) (Grossi, 1895; Laval, 2014; Cárdenas, 2017). El Dr. Grajales también practicó las disecciones anatómicas en el Hospital San Francisco de Borja (HSFB) (Laval, 2014; Cárdenas, 2017). Antes de la apertura de la Escuela Médica del IN, el Dr. Pedro Morán (chileno 1771 - 1839, cirujano y médico) y el Dr. Cox, abrieron una Escuela Privada para médicos y cirujanos, donde se enseñaba anatomía, patología y cirugía, en los hospitales disponibles (Salas, 1894; Benavente; Ferrer; Laval, 1964).

Tercera etapa (1833-1839). Desde la inauguración de la EM33 y hasta la muerte del Dr. Pedro Morán en 1839: esta primera escuela de medicina de Chile independiente estaba dividida en 2 establecimientos, lo teórico se llevaba a cabo en el IN y lo práctico en el HSJD (Pérez, 1993). El Dr. Morán hacia practicar a los estudiantes la disección de cadáveres, para conocer sus órganos y ejercitándose primero como disectores y demostrando después como maestros (OrregoLuco, 1922) (Fig. 3). El Dr. Morán basaba su curso en libros franceses (Chaussier, Bichat, Maygrier y Tiedemann) (Tezanos, 1989; Silva et al., 2019, 2020). Para la EM33, en el HSJD se habilitó el primer Anfiteatro Anatómico (AA). Éste estaba ubicado en el primer patio del hospital, se componía de 2 piezas de adobe, bajas, mal ventiladas y mal iluminadas. Con una puerta y una ventana cada una. En cuanto a los útiles necesarios, no estaba mejor puesto, unas cuantas navajas como escalpelos, un serrucho, martillos, un escoplo y mesas con cubiertas de mármol (Salas; Orrego-Luco). El Dr. Miguel Semir (chileno 1832 - 1882, médico) indica, que "había que tener una abnegación de sí mismo o tener una inspiración divina para poder trabajar en anatomía cuando uno se presentaba por primera vez a presenciar el asqueroso cuadro del anfiteatro y ver destrozos de los miembros humanos, cuya putridez se hallaba encerrada en el mal cuarto en que se verificaba la disección, sin aire que lo ventilase, sin agua, ni paños con que asearse, sin un vestuario adecuado para cubrir el cuerpo de los alumnos y sin ninguna regla higiénica que precaviese de los funestos estragos de la putrefacción y los contagios" (Semir, 1860; Cárdenas \& Mora, 2018). Aún las disecciones se realizaban en invierno. En 1834, llega a Chile el Dr. Laurent Sazié (francés 1807 1865, cirujano y médico), y es nombrado Profesor de Cirugía y Obstetricia (Fig. 4). En 1835 efectuó las primeras disecciones anatómicas en fetos, con el fin de iniciar los estudios obstétricos a sus primeros alumnos (de matronería y medicina). A la muerte del Dr. Morán, es nombrado el Dr. Sazié Profesor Auxiliar de Anatomía (Benavente; Flores; Cárdenas-Valenzuela, 2020).

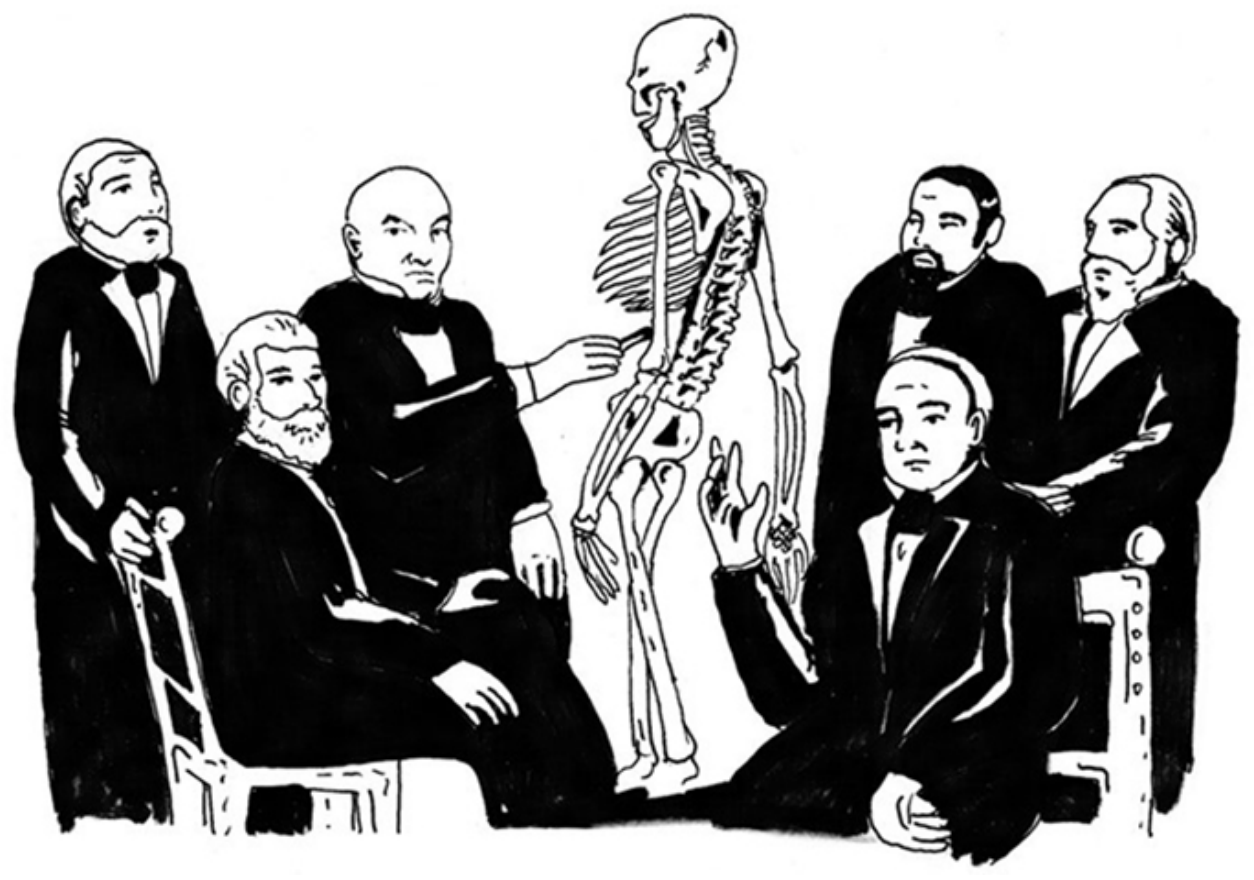

Fig. 3. El Dr. Pedro Morán acompañado de sus estudiantes en la EM33. (representación de los autores según relatos históricos, basado en "La Lección de Anatomía del Dr. Sebastian Egbertsz", obra de Thomas de Keyser, 1619). 


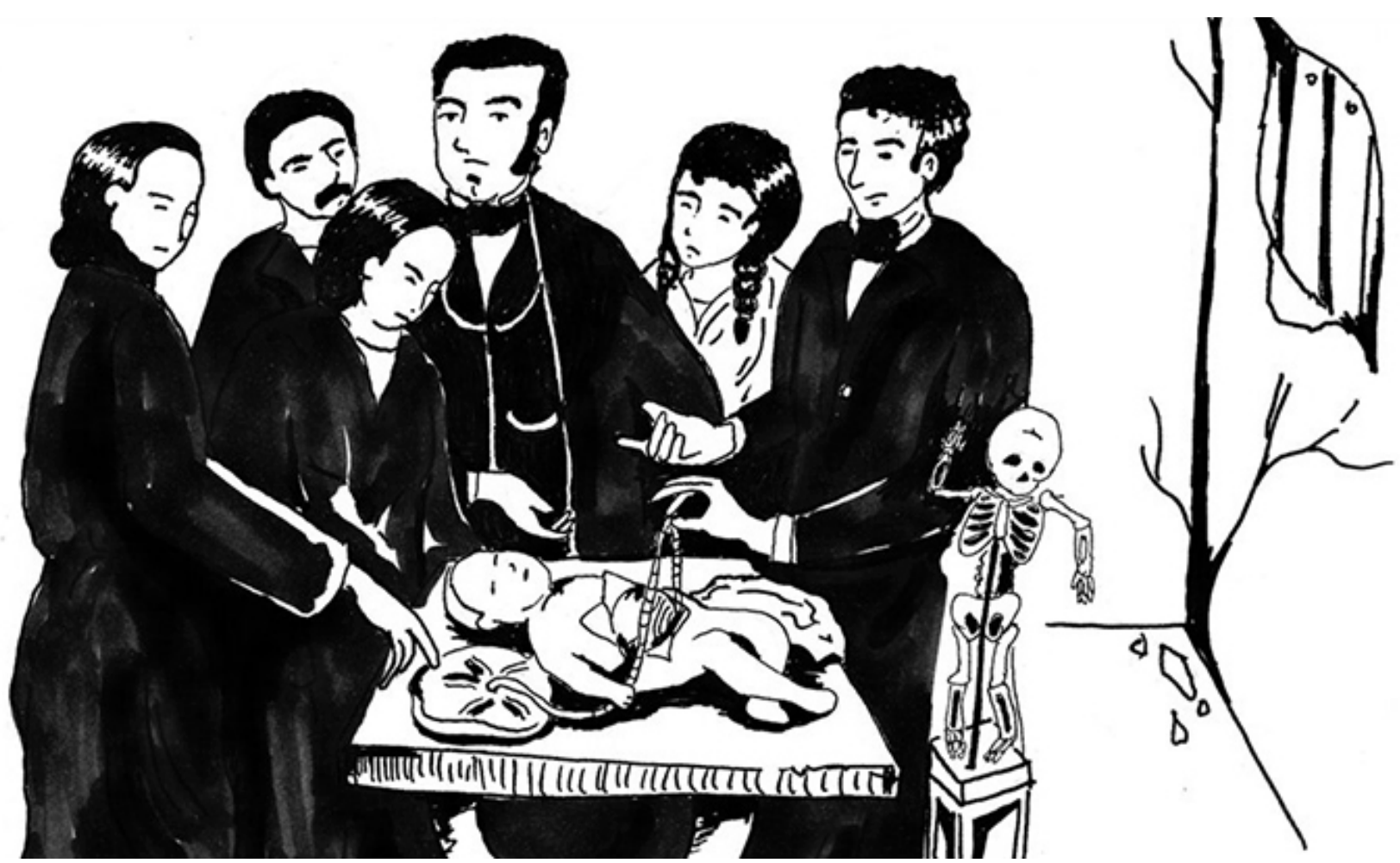

Fig. 4. El Dr. Laurent Sazie, preparando matronas en el HSFB. A estas clases se sumaban estudiantes de medicina (representación de los autores según relatos históricos, basado en "La Lección de Anatomía del Dr. Frederyck Ruysh", obra de Jan Van Neck, 1683).

Cuarta etapa (1839-1850). Desde el nombramiento del Dr. Lafargue como Profesor de Anatomía en 1842 hasta el nombramiento como Profesor de Anatomía del Dr. José Joaquín Aguirre en 1850: posterior a la muerte de Morán y al interinato en la Cátedra de Anatomía por Bartolomé Morán (chileno $i-$ ?, estudiante de medicina y cirujano naval), le sucede en 1842, el Dr. François Jules Lafargue (francés i1850, médico) (Fig. 5). La anatomía bajo la tutela de este profesor se enseñaba en forma práctica y teórica. A una excelente disección, agregaba una disertación, de manera que se revisaba la anatomía descriptiva y topográfica al mismo tiempo. El Dr. Lafargue habría puesto sus clases a la altura de las clases francesas y en palabras del Dr. Aguirre, las preparaciones que presentaba a sus alumnos eran ejecutadas con una asombrosa habilidad (Salas; Laval, 1964; Alonso, 1970). En 1843, queda vacante la Cátedra de Anatomía. Reemplazaron a Lafargue, el Dr. Víctor Nicolas Pretot Meslhier (francés 1818 - 1867, médico) y el Dr. Vicente Antonio Padín del Valle (chileno 1815 - 1865, médico), quienes limitaron la clase a las disecciones de los alumnos y a la lección del profesor. Los textos guía de este periodo, fueron el "Manual Anatómico del Disector" de Alejandro Lauth (1841) y el "Tratado de Anatomía Descriptiva" de Jean Cruveilhier (1834 - 1836) (Salas; Laval, 1964; Cárdenas-Valenzuela). En 1844, las condiciones del AA seguían siendo tan míseras como en 1833. Eran los estudiantes los que debían hacer el aseo y transportar los cadáveres (Vicuña-Mackenna, 1944). Recién en 1847, Antonio Varas de la Barra (chileno 1817 - 1886, abogado y político), Rector del IN, pedía nombrar un sirviente para estos menesteres (Orrego-Luco). Y en 1856, se nombró un Profesor Auxiliar o Disector (Salas), cargo que ocupó el destacado estudiante, Adolfo Valderrama Sainz de la Peña (chileno 1834 - 1902, médico).

Quinta etapa (1850-1889). Desde el nombramiento del Dr. José Joaquín Aguirre como Profesor de Anatomía en 1850 hasta la inauguración de la Escuela Médica de la Cañadilla en 1889: el Dr. José Joaquín Aguirre Campos (chileno 1822 - 1901, médico), incluyó importantes cambios al curso (Fig. 6). Reemplazó el texto guía por el "Nouveau Traité Elementaire D'Anatomie Descriptive" de Jean Alexandre Jamain (1853), usado en Francia. Promovió que los estudiantes prepararan y presentaran sus disecciones anatómicas en clases (Laval, 1964). Este significativo cambio, hizo que fuera más práctico y enriquecedor el estudio de la asignatura (Orrego-Luco). Y logra demostrar que la disección anatómica es un instrumento para aprender una disciplina de trabajo y conseguir un método activo de aprendizaje (Alonso). En 1857, se establece un segundo AA (la "pesebrera") en un caserón inmediato al HSJD. Este AA comprendía 2 habitaciones. En la primera, utilizada por el 


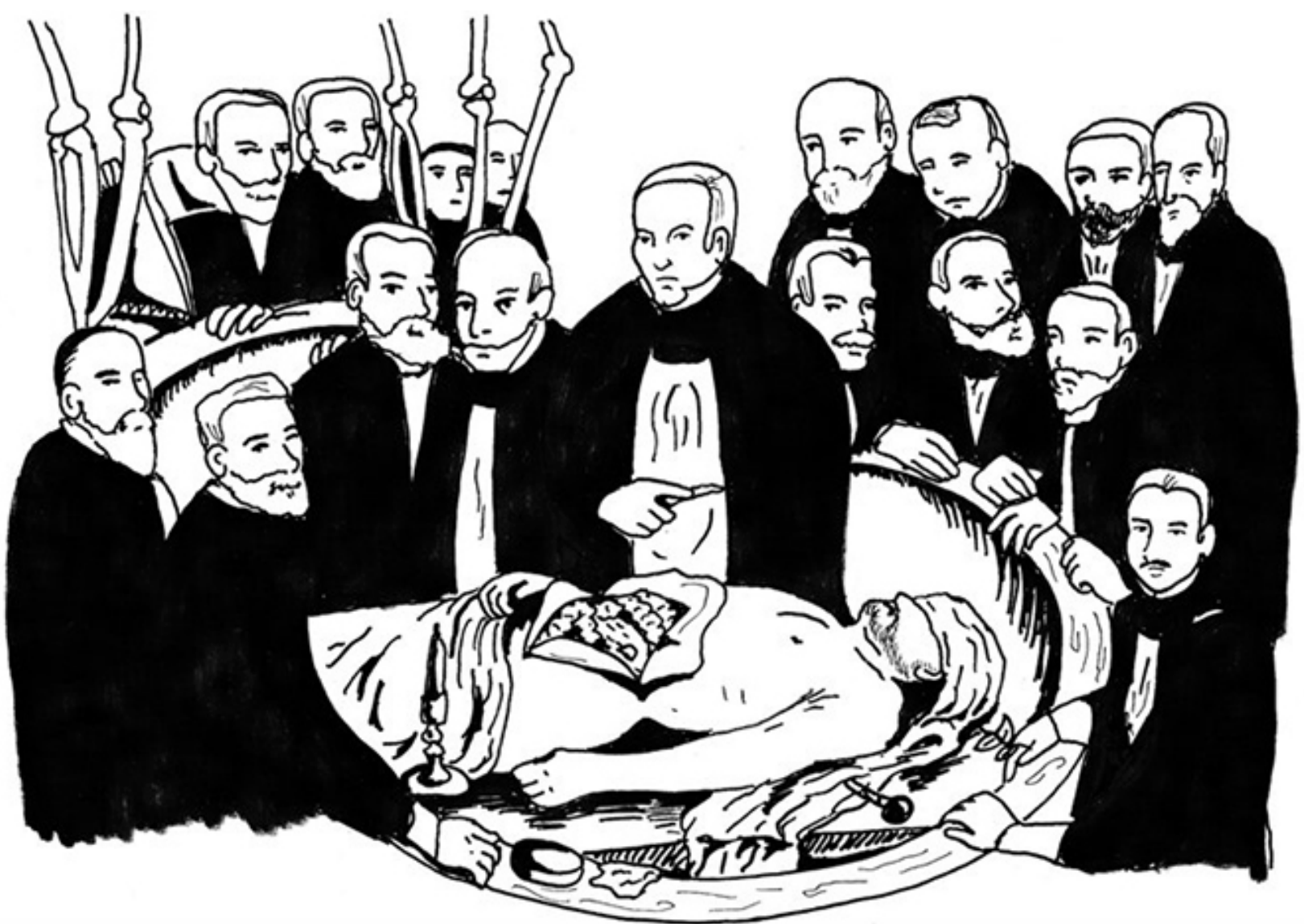

Fig. 5.- El Dr. François Julien Lafargue, en sus labores de disección anatómica para la enseñanza de la anatomía. Estas clases se volvieron muy concurridas por los médicos de la ciudad, que acompañaban a los estudiantes de medicina en las prácticas de este profesor (representación de los autores según relatos históricos, basado en "La Lección de Anatomía del Dr. Willem Van Der Meer", obra de Michael Jansz Van Mierevelt, 1617).

Profesor y el Disector, había una claraboya, una mesa de mármol, un armario para instrumentos y unos cuantos bancos. La segunda era para los estudiantes, no tenía luz y para poder trabajar en sus disecciones, los estudiantes sacaban las mesas y las colocaban bajo una mediagua, sin más amparo que el techo de zinc. En esa intemperie, se sentía frio en el invierno y para no pisar en el suelo húmedo se ponían ladrillos. Naturalmente se mojaban cuando llovía y se veían obligados a disecar, protegiéndose con un paraguas amarrado al tórax (Orrego-Luco; Maffet, 1939). Debido a las exigencias del curso del Dr. Aguirre, de disecar una gran cantidad de preparaciones anatómicas, los alumnos se veían obligados a obtener cadáveres de la Morgue de Santiago (Sotomayor \& Délano, 1922; León, 1997). En 1859, Don Ignacy Domeyko Ancuta (polaco 1802 - 1889, científico) decía, "quédame por recordar lo pobre y limitada que se halla en sus recursos la Facultad de Medicina... carece también esta enseñanza de un local aparente para los estudios, de un anfiteatro de disecciones conveniente, de salas organizadas a propósito para la clínica tanto externa como inter- na y de colecciones anatómicas y patológicas" (Salas). Pese a las evidentes precariedades, las nuevas dependencias habilitadas en la calle de San Francisco se inaugurarían como la nueva Escuela Médica en 1863 (Orrego-Luco; Maffet), y no hubo cambios significativos en estas nuevas dependencias hasta la inauguración de la Escuela Médica de la Cañadilla en 1889 .

\section{CONCLUSIÓN}

El análisis del periodo en cuestión permite evidenciar la pobreza, precariedad y humildad en que se inició y desarrolló la enseñanza de la anatomía como base de la formación médica en Chile. A pesar de esta situación la Escuela Médica de 1833 logró sobreponerse y continuar su ardua tarea histórica. Todos los profesores de la asignatura contaban con una buena preparación, fueron muy queridos por los estudiantes y recordados con cariño. Pusieron todo de su 


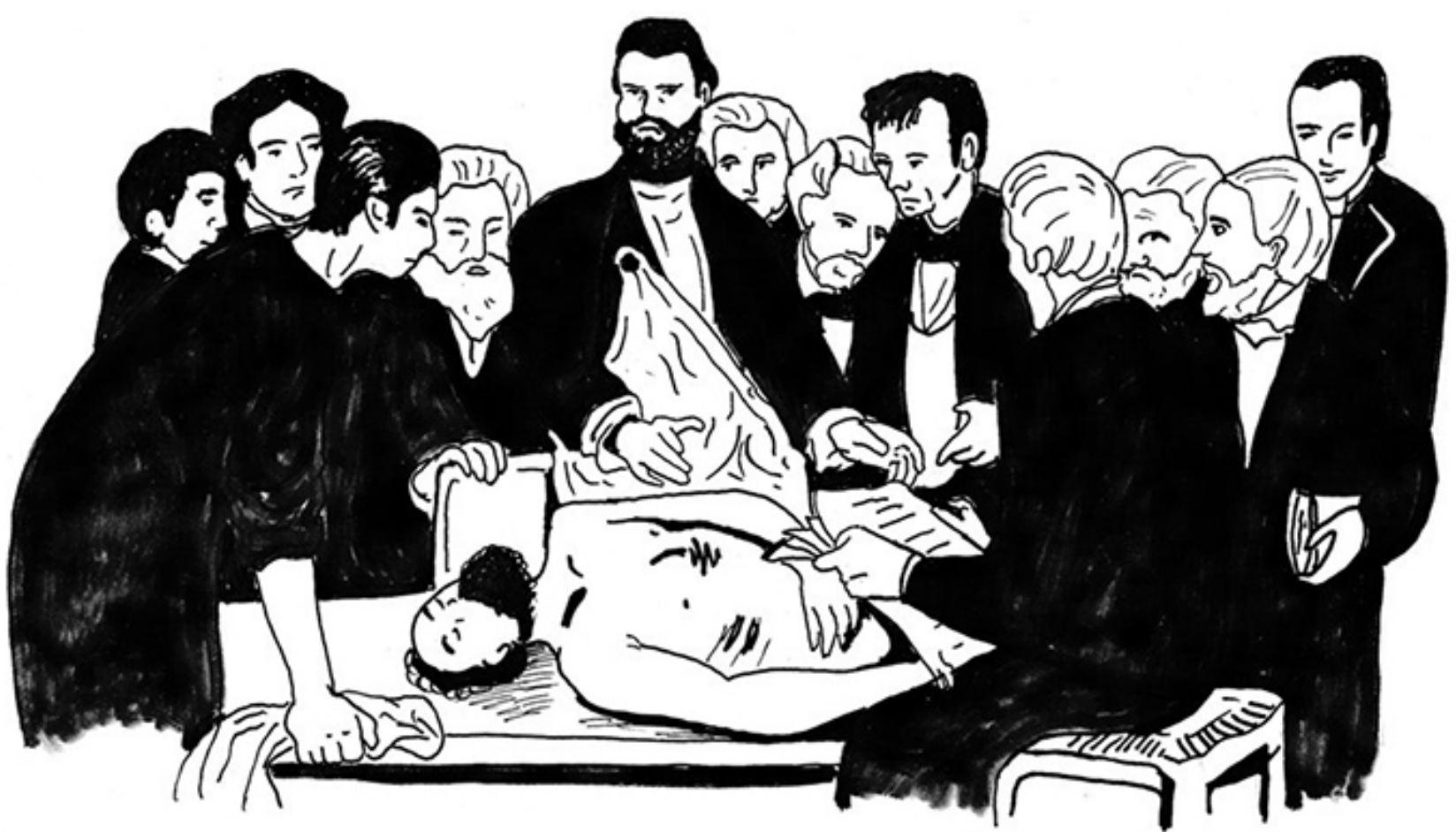

Fig. 6. El Dr. José Joaquín Aguirre, en sus labores de la enseñanza de la anatomía (representación de los autores según relatos históricos, basado en "La Lección de Anatomía del Dr. Velpeau en la Charité", obra de Augustine Ferret-Perrin, 1864).

parte, aportaron con conocimientos y muchas veces con su propio peculio. La mayoría de su práctica tomó como base la influencia europea, pero debió adaptarse a las condiciones físicas y humanas disponibles. Pese a los esfuerzos del estado, esta realidad no cambió hasta la Escuela Médica de 1889 y estas primeras generaciones pagaron, muchas veces con su vida, el interés de ser los primeros médicos para la República de Chile.

SILVA, J.; ARAYA, C.; CÁRDENAS, J.; MANSILLA, B.; SALAZAR, M. \& LATIN, A. First anatomical dissections for teaching purposes in Chile. Int. J. Morphol., 39(1):143-150, 2021.

SUMMARY: In Chile, the first teachings of anatomy were carried out in the XVI century, to develop the knowledge of health profesionals practicing in the country. In 1704, Pope Sixtus IV allowed the practice of dissection, led by city's mayor of the city and where doctors and surgeons were invited. Subsequently, the exclusively theoretical teaching of medicine at the Real Universidad de San Felipe encouraged teachers to teach anatomy in clinical and practical ways to complement the lectures. Independence of Chile, found that university careers suffer major curricular changes, although in medicine and anatomy, development was slow and tortuous. The aim of this study was to describe the practice of anatomical dissection in training doctors in Chile during the period between 1700 and 1889. A literature review was conducted in scientific articles related to the subject and books on the history of Chile and the history of Chilean medicine. This allowed us to des- cribe the beginnings, development and characteristics of the dissection practice used in the training of physicians in the period in question. The analysis of the results allowed us to identify 5 different stages, strongly influenced by their historical context and differentiated by the approach used by the teachers of each of these stages, who defined a bibliography, an orientation and a dissection practice. In turn, the focus of each teacher was based on the academic training of each of them. This work allowed ordering and exposing valuable information for the history of medicine in Chile, as well as understanding the educational practices that were carried out in the training of these professionals who had a critical role in the process of this developing nation.

KEY WORDS: History of Chile; History of medicine; History of anatomy; Anatomical dissection.

\section{REFERENCIAS BIBLIOGRÁFICAS}

Alonso, A. Vida y obra del Dr. José Joaquín Aguirre y la medicina Chilena de la época. An. Chil. Hist. Med., 12(1):33-74, 1970.

Benavente, R. El Protomedicato en Chile. Santiago de Chile, Soc. Imp. y Lit. Universo, 1928.

Cárdenas, J. El Instituto de Anatomía de la Universidad de Chile. An. Chil. Hist. Med., 16(1):33-42, 2006.

Cárdenas, V. J. L. History of anatomy in Chile. The beginnings. Int. J. Morphol., 35(3):958-69, 2017.

Cárdenas, J. \& Mora, D. La infección cadavérica en Chile, muerte en el anfiteatro anatómico. Rev. Chil. Infectol., 35(5):595-600, 2018. 
Cárdenas-Valenzuela, J. History of anatomy in Chile part II. The Alma Mater. Int. J. Morphol., 38(4):1074-89, 2020.

Ferrer, P. Historia General de la Medicina en Chile. Desde el Descubrimiento y Conquista de Chile en 1535 , hasta nuestros días. Talca, Imprenta Talca, 1904.

Flores, N. Historia de la anatomía en Chile. Arch. Chil. Morfol., 1:7-25, 1933.

Grossi, J. Reseña del Progreso Médico en Chile. Valparaíso, Imprenta de La Opinión, 1895.

Laval, E. Evolución y desarrollo de la enseñanza de la anatomía en Chile. An. Chil. Hist. Med., 6(2):7-75, 1964.

Laval, E. Manuel Julián Grajales. Propagador de la vacuna antivariólica en América del Sur. Anatomista y cirujano. Rev. Chil. Infectol., 31(6):7435, 2014.

Laval, E. \& Duarte, I. Enseñanza de la medicina en Chile colonial durante el siglo XVIII. El catedrático Domingo Nevín y su alumno Pedro Manuel Chaparro. Rev Chil. Infectol., 33(5):565-9, 2016.

León, M. Sepultura Sagrada, Tumba Profana. Los Espacios de la Muerte en Santiago de Chile, 1883 - 1932. Santiago de Chile, LOM, 1997.

Maffet, H. El cuerpo médico y la medicina en Chile. Santiago de Chile, Imprenta Chile, 1939.

Medina, J. La Medicina y los Médicos de la Real Universidad de San Felipe. Santiago de Chile, Soc. Imp. y Lit. Universo, 1928.

Neghme, A. Panorama de la Educación Médica en Chile. Santiago de Chile, Ediciones de la Academia de Medicina del Instituto de Chile, 1988.

Orrego-Luco, A. Recuerdos de la Escuela. Santiago de Chile, Imprenta Universitaria, 1922.

Pérez, J. La Escuela de Blest, Sazié y Aguirre 1833 - 1993. Santiago de Chile, Editorial Universitaria, 1993.

Salas, E. Historia de la Medicina en Chile. Santiago de Chile, Imprenta Vicuña Mackenna, 1894.

Semir, M. Apuntes para la historia de la enseñanza de la medicina en Chile. An. Univ. Chile, 17(8):737-56, 1860.

Silva, J.; Araya, C.; Cárdenas, J.; Salcedo, A.; Mansilla, B.; Hernández, A.; Rodriguez, P.; Salazar, M.; Caracuel, R. \& Espinoza, M. Anatomical bibliography used in the training of physicians in Chile Between 1758 and 1840. Int. J. Morphol., 37(3):938-46, 2019.

Silva, J.; Araya, C.; Cárdenas, J.; Mansilla, B.; Latín, A. \& Salazar, M. First anatomical atlas used in the training of physicians in Chile. Int. J. Morphol., 38(5):1302-10, 2020.

Sotomayor, A. \& Délano, J. Un Establecimiento que es el Segundo en el Mundo, el Instituto de Medicina Legal. Santiago de Chile, Revista Sucesos, 1057, 1922.

Tezanos, S. Semblanza del Dr. Pedro Moran. Jornadas de Historia de la Medicina Dr. Amador Neghme R. Santiago, 1989.

Vicuña-Mackenna, B. Los Médicos de Antaño en el Reino de Chile. Santiago de Chile, Editorial Difusión, 1944.
Dirección para correspondencia:

Prof. Dr. Juan Luis Silva R.

Facultad de Medicina y Ciencia

Universidad San Sebastián

Lota 2465. C. P. 7510157

Providencia

Santiago

CHILE

Email: juan.silva@uss.cl

Recibido : 17-09-2020

Aceptado: 20-10-2020 